\title{
Mitotic catastrophe triggered in human cancer cells by the viral protein apoptin
}

\author{
HL Lanz ${ }^{1}$, RME Zimmerman ${ }^{1}$, J Brouwer ${ }^{1}$, MHM Noteborn ${ }^{1}$ and C Backendorf ${ }^{*, 1}$
}

Mitotic catastrophe is an oncosuppressive mechanism that senses mitotic failure leading to cell death or senescence. As such, it protects against aneuploidy and genetic instability, and its induction in cancer cells by exogenous agents is currently seen as a promising therapeutic end point. Apoptin, a small protein from Chicken Anemia Virus (CAV), is known for its ability to selectively induce cell death in human tumor cells. Here, we show that apoptin triggers p53-independent abnormal spindle formation in osteosarcoma cells. Approximately $50 \%$ of apoptin-positive cells displayed non-bipolar spindles, a 10 -fold increase as compared to control cells. Besides, tumor cells expressing apoptin are greatly limited in their progress through anaphase and telophase, and a significant drop in mitotic cells past the meta-to-anaphase transition is observed. Time-lapse microscopy showed that mitotic osteosarcoma cells expressing apoptin displayed aberrant mitotic figures and/or had a prolonged cycling time during mitosis. Importantly, all dividing cells expressing apoptin eventually underwent cell death either during mitosis or during the following interphase. We infer that apoptin can efficiently trigger cell death in dividing human tumor cells through induction of mitotic catastrophe. However, the killing activity of apoptin is not only confined to dividing cells, as the CAV-derived protein is also able to trigger caspase-3 activation and apoptosis in non-mitotic cancer cells.

Cell Death and Disease (2013) 4, e487; doi:10.1038/cddis.2013.2; published online 7 February 2013

Subject Category: Cancer

Mitotic catastrophe is an oncosuppressive mechanism emerging as a promising end point in cancer treatment. ${ }^{1,2}$ Originally used as a denomination for 'death during mitosis', mitotic catastrophe reached a tentative consensus, defined as a mechanism that senses mitotic failure resulting in an irreversible fate, such as apoptosis, necrosis or senescence. The aberrant mitosis can be caused either by premature or inappropriate entry into mitosis or a failure of related checkpoints in combination with cellular damage. ${ }^{2,3}$ Mitotic catastrophe is considered an oncosuppressive pathway due to its association with absent or weakened checkpoints. ${ }^{4}$ It has been argued that the evasion of mitotic catastrophe constitutes one of the gateways to cancer development. ${ }^{2}$

Apoptin is a small avian-virus-derived protein capable of selectively inducing apoptosis in human tumor cells, whereas having no detrimental effect on healthy normal cells. ${ }^{5,6}$ In normal cells, it localizes to the cytoplasm where it is efficiently degraded by the proteasome. ${ }^{7}$ On the contrary, in transformed cells, apoptin is phosphorylated on threonine 108 and localizes to the nucleus. ${ }^{8}$ Until now, apoptin has been shown to induce apoptosis in over 70 human cancer cell lines, ${ }^{5}$ and in preclinical mouse model studies apoptin acted as an efficient and safe anticancer therapeutic. , $10^{-10}$

Recently, we showed that the tumor-selective behavior of apoptin can be triggered in normal human fibroblasts by downregulation of the $\mathrm{B} 56 \gamma$ or $\mathrm{B} 56 \delta$ subunits of the tumor-suppressor protein phosphatase $2 \mathrm{~A}$ (PP2A). ${ }^{11}$ The B56-PP2A complex is enriched at the centromeres and centrosomes of mitotic cells, and is responsible for the formation of stable attachments between kinetochores and microtubules. ${ }^{12}$ Inactivation of PP2A by viral oncoproteins contributes to cell transformation ${ }^{13}$ possibly by increasing the frequency of chromosomal instability. ${ }^{12}$ These findings raised the question whether apoptin can sense chromosomal instability and perturb spindle formation during mitosis.

\section{Results}

Apoptin-dependent abnormal spindle formation is independent of p53. We first examined the impact of ectopically expressed apoptin on the mitotic process in human osteosarcoma cells (either Saos-2: p53-null cells ${ }^{14}$ or U2Os: p53wild-type cells ${ }^{15}$ ). Cells were transiently transfected with plasmid encoding either flag-apoptin or flag-SPRR4, a skin cornification protein ${ }^{16}$ that was used as a control and does not induce apoptosis in human cells (Supplementary Figure S1). Both apoptin-positive and apoptin-negative mitotic cells within the same transfected dish were scored for normal/abnormal bipolar spindle formation. The data demonstrate that apoptin expression caused an increase in abnormal spindle formation in mitotic cells that was not observed in non-transfected cells in the same dish or in SPRR4-transfected cells (Figures $1 \mathrm{a}$ and $\mathrm{h}$ ). No differences were observed between Saos-2 (Figures 1a-g) and U2OS cells (Figures $1 \mathrm{~h}-\mathrm{n}$ ). Apparently, the effect exerted by apoptin during mitosis in cancer cells is not dependent on

\footnotetext{
${ }^{1}$ Department of Molecular Genetics, Leiden Institute of Chemistry, Leiden University, Leiden, The Netherlands

*Corresponding author: C Backendorf, Department of Molecular Genetics, Leiden Institute of Chemistry, Leiden University, Einsteinweg 55, Leiden 2333, The Netherlands. Tel: + 3171 5274409; Fax: + 3171 5274357; E-mail: backendo @ chem.leidenuniv.nl

Keywords: apoptosis; metaphase-anaphase transition; APC/C; non-bipolar spindle; mitotic instability

Abbreviations: Apoptin, apoptosis-inducing protein; APC/C, anaphase-promoting complex/cyclosome; PP2A, protein phosphatase 2A; SAC, spindle assembly checkpoint; GFP, green-fluorescent protein

Received 18.7.12; revised 16.12.12; accepted 27.12.12; Edited by H-U Simon
} 
a
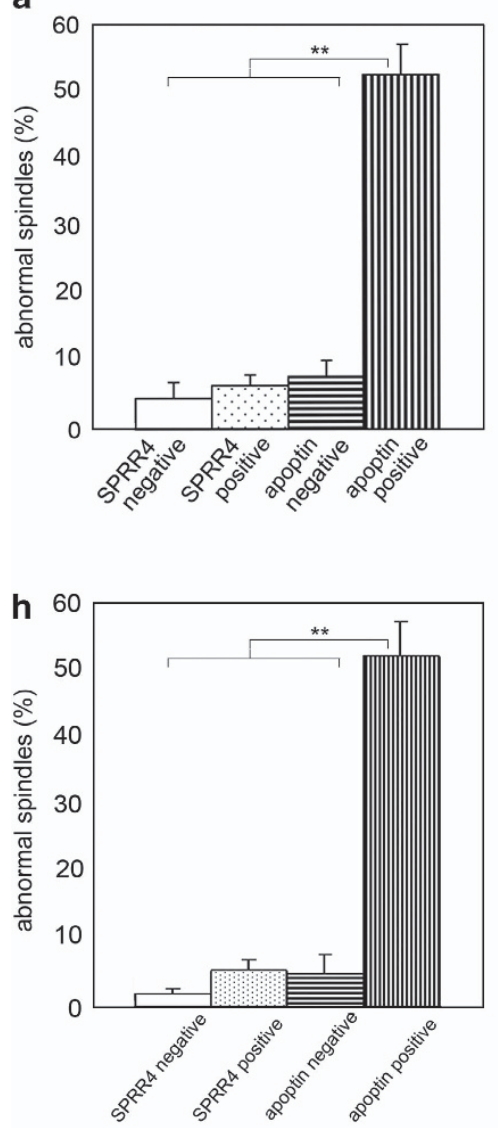

b

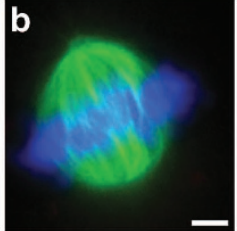

SPRR4 negative

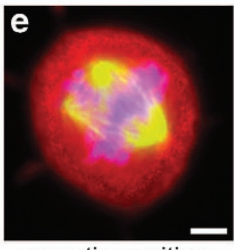

apoptin positive

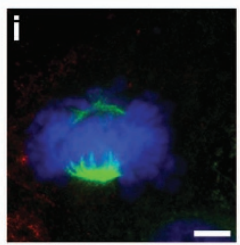

SPRR4 negative

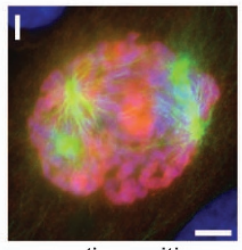

apoptin positive

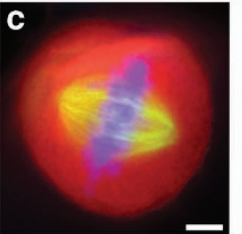

SPRR4 positive

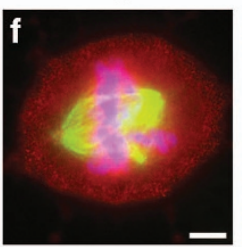

apoptin positive

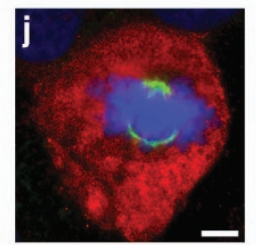

SPRR4 positive

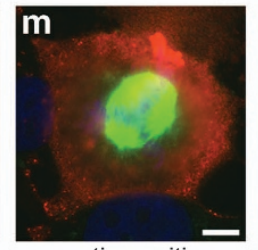

apoptin positive

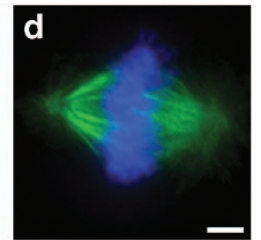

apoptin negative

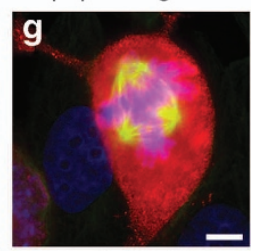

apoptin positive

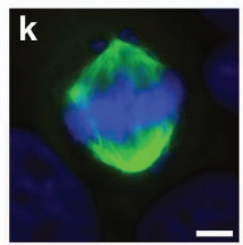

apoptin negative

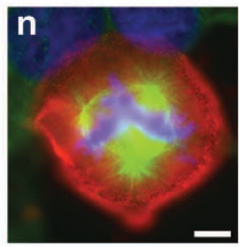

apoptin positive

Figure 1 Abnormal spindle formation in apoptin-expressing Saos-2 or U2OS osteosarcoma cells. Cells were transiently transfected with plasmid encoding flag-apoptin or flag-SPRR4 and fixed 72 or $48 \mathrm{~h}$ post transfection (Saos-2 and U2OS, respectively). Flag-tagged apoptin or flag-tagged SPRR4 were stained with a flag-specific antibody conjugated to rhodamine (red), endogenous tubulin with a FITC-labeled tubulin-specific antibody (green) and DNA with Hoechst (blue). Both transfected and non-transfected cells (in the same dish) were analyzed for spindle formation. Bipolar spindles were scored as normal, all others as abnormal. Data obtained from Saos-2 cells are represented in panels $(\mathbf{a}-\mathbf{g})$ and those from U2OS cells in panels $(\mathbf{h}-\mathbf{n})$. Panels $(\mathbf{a})$ and $(\mathbf{h})$ represent the percentages of abnormal spindle formation from three separate experiments for each cell line (represented as mean \pm S.D.). Per experiment, 100 mitotic cells that were positive or negative for flag-apoptin or flag-SPRR4 were scored. Apoptin-positive mitotic cells contained significantly more abnormal spindles $(P<0.01)$ than the three control groups (respectively, $P=0.004,0.005$ and 0.006 for Sa0s-2 cells, and $P=0.002$, 0.008 and 0.005 for U2OS cells). Representative pictures of normal bipolar spindles in the three control groups: (b, i) non-transfected cells in the SPRR4 experiment; (c, j) SPRR4-expressing cells; (d, k) non-transfected cells in the apoptin experiment. Panels (e-g) and (I-n) show representative pictures of abnormal spindles in apoptinpositive cells. Scale bar: $5 \mu \mathrm{m}$

functional p53, whereas the presence of non-bipolar spindles hints to mitotic catastrophe as the process which is induced. ${ }^{17}$

Apoptin prevents meta-to-anaphase transition. Half of the apoptin-positive mitotic cells showed normal spindle formation during metaphase in both Saos-2 and U2OS cells (Figures 1a and h). To examine whether these bipolarspindle-positive cells progressed through mitosis in a regular fashion, dividing cells were scored according to their respective phases in mitosis: prophase, metaphase, anaphase or telophase. Non-transfected cells displayed all four mitotic phases (Figure 2a). Regular mitotic figures in apoptinpositive cells were, however, mainly restricted to prophase (Figure 2b) and metaphase (Figure 2c), whereas in SPRR4transfected control cells anaphase (Figure 2d) and telophase (Figure $2 \mathrm{e}$ ) cells could readily be found. For quantification purposes, mitotic cells were classified in groups of prophase/ metaphase or anaphase/telophase. In both Saos-2 cells
(Figure 2f) and U2OS cells (Figure 2g), apoptin expression resulted in a significant drop in the percentage of anaphase/ telophase cells $(P=0.0041$ for Saos- 2 and $P=0.0055$ for U2OS cells). No significant difference was found in the distribution of the two mitotic groups in apoptin-positive p53-nul Saos-2 and p53-wt U2OS cells $(P=0.38)$. Apparently, even in those cases where spindles appear normal during the metaphase in apoptin-expressing cells, cells are not able to easily progress past the spindle assembly checkpoint (SAC) into anaphase. Again, this effect did not depend on functional $\mathrm{p} 53$.

Apoptin-positive cells undergo cell death during cell division. During our analysis, dying cells were observed in which tubulin staining indicated that apoptin-induced cell death had occurred during the mitotic cell cycle stage. These include several cells with membrane blebbing, DNA fragmentation and clear spindle remnants (Figure 2h), an 

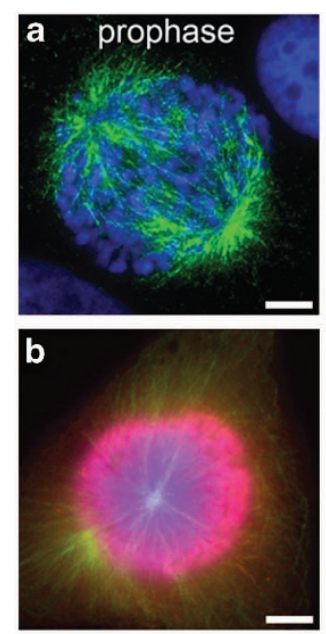

apoptin positive prophase

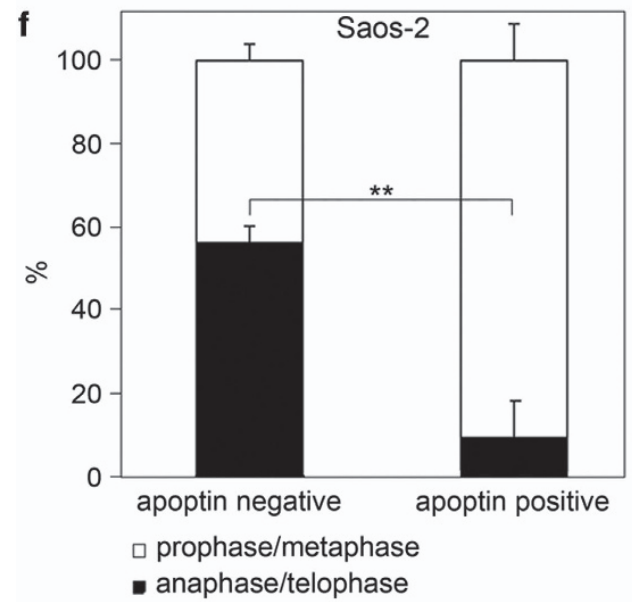

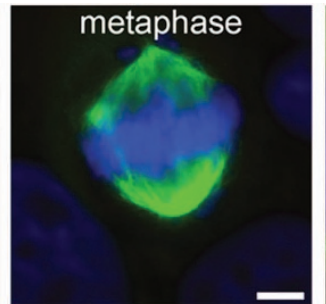

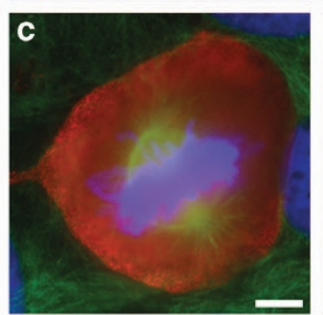

apoptin positive metaphase
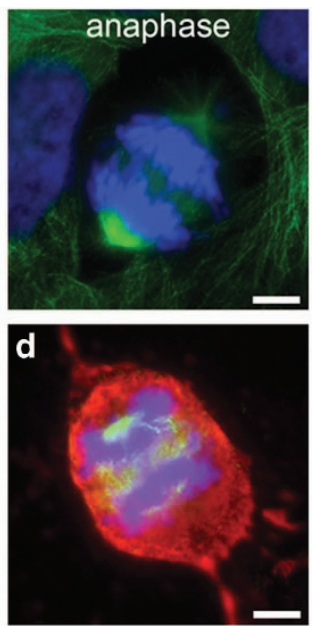

SPRR4 positive anaphase
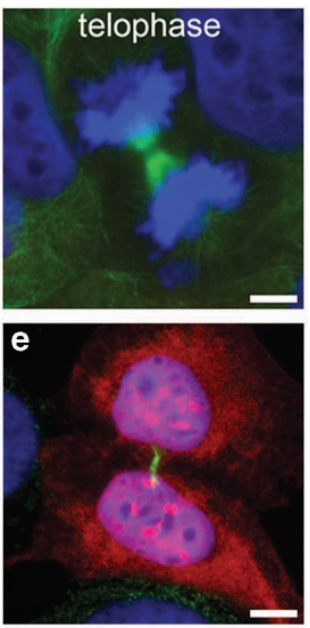

SPRR4 positive telophase
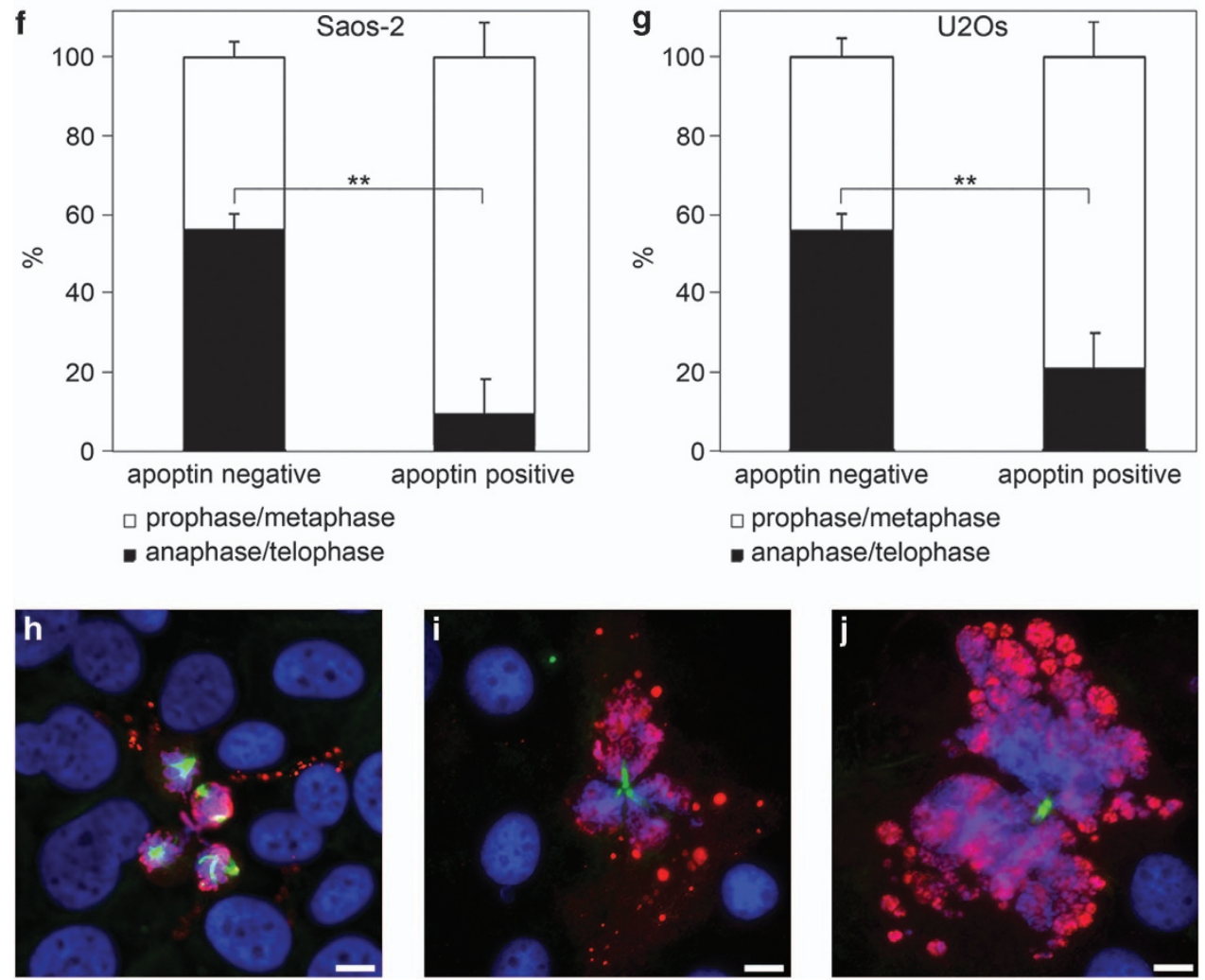

Figure 2 Apoptin inhibits mitotic cells from progressing through anaphase and telophase. U2OS and Saos-2 cells were transiently transfected with plasmid-encoding flagSPRR4 or flag-apoptin, and processed for immune fluorescence analysis as described in the legend of Figure 1. Both flag-apoptin-transfected and untransfected mitotic cells were scored according to their phase in mitosis. Representative images of the four phases in U2OS are in (a) from left to right prophase, metaphase, anaphase and telophase. Apoptin-positive mitotic U2OS cells could be found in prophase (b), and metaphase (c), but were scarce in anaphase and telophase, whereas SPRR4-positive mitotic U2OS cells were readily found in anaphase (d), and telophase/cytokinesis (e). Apoptin transfected mitotic cells were scored as described above, grouped as prophase/metaphase and anaphase/telophase and compared to untransfected cells in the same experiment. The results from three separate experiments (represented as means \pm S.D.) in which 100 mitotic cells per group were counted are given. Panel (f): Saos-2 cells $\left.{ }^{* \star} P=0.0041\right)$. Panel $(\mathbf{g})$ : U2OS cells $\left({ }^{* *} P=0.0055\right)$. The distribution of 'pro/meta' and 'ana/telo' phases did not significantly differ between apoptin-positive p53-wt U2OS and p53-null Saos-2 cells ( $P=0.38$ in a paired two-tailed $t$-test). Scale bar in panels (a-e): $5 \mu$ m. Panels (h-j): apoptin-positive cells are shown that undergo cell death during mitosis: (h) after spindle formation, (i) during abnormal cytokinesis, and (j) during normal cytokinesis. Note membrane blebbing and DNA fragmentation in the dying cells. Scale bar: $10 \mu \mathrm{m}$

apoptin-positive mitotic cell with three spindles that has undergone cell death during telophase showing excessive blebbing (Figure 2i) and finally an apoptin-positive cell that during earlier steps in mitosis would have been classified as normal, but now shows apoptotic blebbing and DNA fragmentation during cytokinesis (Figure 2j). 
Apoptin triggers mitotic catastrophe in osteosarcoma cells leading to cell death. In order to firmly establish that mitosis is disturbed (at various stages) in apoptin-positive cells and in order to determine the extent and timing of this effect, we performed live-cell-imaging by using fluorescent time-lapse videomicroscopy ${ }^{18}$ of U2OS (Figures $3 a-c$ ) or Saos-2 cells (Figure $3 d$ ) transiently transfected with plasmidencoding mCherry-tagged apoptin (red fluorescence) and GFP-tagged tubulin (green fluorescence). Cells that were only positive for GFP-tubulin divided with similar kinetics as untransfected cells, in $\sim 1 \mathrm{~h}$ (Figure $3 \mathrm{a}$ ). An apoptin-expressing cell with three spindles eventually generated daughter cells undergoing cell death during cytokinesis (Figure $3 b$ ). Note that the different phases are prolonged as compared to the cells only expressing GFP-tubulin (compare panel A with panel B). For example, prophase lasts $4.5 \mathrm{~h}$ in the apoptinpositive cell compared to $12 \mathrm{~min}$ in the control, and the entire mitosis took over $11 \mathrm{~h}$ instead of $1 \mathrm{~h}$. Next, an apoptinpositive cell starts to undergo regular mitosis with the formation of two centrosomes and two spindles (Figure 3c). Notice that prophase is delayed to such an extent that an untransfected cell (white circle) completes an entire round of mitosis during the same time. Division is halted during metaphase, and after a block of several hours the cell presents membrane blebbing and DNA fragmentation. An apoptin-expressing Saos-2 cell undergoes a round of seemingly normal mitosis, that is, the cell generates two daughter cells. However, phases are prolonged and in the interphase following division both daughter cells undergo DNA fragmentation (Figure 3d). Importantly, in all apoptinpositive mitotic cells monitored during our analysis, we were able to detect cell death either during mitosis or during the following interphase.

Is apoptin-mediated cell death confined to mitotic cells? The data presented so far clearly indicate that mitotic cells are very efficiently killed by apoptin. This raised the question whether mitotic catastrophe is the only cause of cell death in apoptin-transfected cell cultures, or whether apoptin can also induce apoptosis in interphase cells. In order to answer this question, we have arrested cell cycle progression in apoptin-transfected U2OS or Saos-2 cultures by serum starvation and mimosine treatment. Under these conditions, cells are blocked in the G1/G0 phase of the cell cycle (Figure 4a). Interestingly, in such arrested cultures, apoptin expression resulted in a consistent drop in viability in both U2OS and Saos-2 cells (Figure 4b, black bars), which does not significantly differ from its effect on control cycling cells (white bars). No significant difference was found between apoptin-positive cycling and arrested cells, which was likely due to the fact that in transiently transfected cycling cultures, the percentage of mitotic cells was rather low (2-4\% at the time of analysis). Cell death in arrested cells can be classified as apoptosis as it is paralleled by caspase-3 activation (Figure 4c). Mock-transfected cells or cells transfected with SPRR4 do not show a drop in survival, neither under cycling nor cell cycle-arrested conditions (Figure 4b). Apparently, apoptin can also efficiently induce apoptosis in non-cycling cells arrested in G1/G0.

\section{Discussion}

Our analysis proves that in mitotic apoptin-expressing tumor cells, various well-defined events occur in a clearly successive manner. These include perturbed spindle formation, slower progression through the various phases of mitosis and eventually induction of cell death. Therefore, the observed behavior of a mitotic cancer cell expressing apoptin conforms to the set of characteristics attributed to mitotic catastrophe by the 2012 Nomenclature Committee on Cell Death: (a) it is triggered by perturbations of the mitotic apparatus; (b) it is initiated during the $\mathrm{M}$ phase of the cell cycle; (c) it is paralleled by some degree of mitotic arrest; (d) it ultimately triggers cell death or senescence. ${ }^{1}$

We have recently shown that apoptin can be activated in normal cells by inactivation of the B56 $\gamma$ or $\delta$ subunits of PP2A. ${ }^{11}$ The PP2A-B56 complex is essential for the stabilization of kinetochore-microtubule attachments and has an important role in correct chromosome segregation. ${ }^{12}$ Similarly, knockdown of the SAC kinase Bub1 can trigger tumor-like behavior of apoptin in normal cells. ${ }^{19}$ Apparently, apoptin can sense malfunctioning of the SAC. In the present study, both the timing of initiation (at the meta-to-anaphase transition) and the character of the various mitotic perturbations that are observed also pinpoint at an activation of apoptin around the mitotic-spindle checkpoint. As many (if not all) human tumor cells have a weakened $S A C,{ }^{20}$ our analysis implies that apoptin is likely able to sense minor inaccuracies of the SAC in tumor cells in a similar way as previously shown in genetically modified normal cells. ${ }^{11,19}$

How then can active apoptin induce mitotic instability and cell death? As long as spindles are not correctly organized and not all kinetochores are properly attached, the SAC is activated and prevents progression through mitosis by directly signaling to the anaphase-promoting complex/cyclosome (APC/C) ${ }^{21}$ Inhibition of APC/C by the SAC leads to stabilization of cyclin B1, and prolonged activation of cyclin B1 is associated with mitotic catastrophe. ${ }^{22}$ In this respect, it is relevant to mention that increased levels of cyclin B1 have actually been observed following apoptin expression in cancer cells. ${ }^{23}$ Additionally, it has been shown that apoptin is able to associate directly with APC1, a subunit of the APC/C, and that the resulting inactivation of $\mathrm{APC} / \mathrm{C}$ is sufficient for the induction of apoptosis. ${ }^{23}$

By combining the cellular analysis presented here with the earlier molecular evidence mentioned above, the following model of apoptin action arises. Apoptin senses a weakened $S A C^{11,19}$ and binds directly to APC1 that is dislodged from the APC/C complex. ${ }^{23}$ Inhibition of APC/C results in a serious disabling of the mitotic checkpoint control, resulting in blockage of the meta-to-anaphase transition (Figures $2 f$ and g) and the appearance of atypical non-bipolar spindles (Figure 1). Cell death can be induced either in cells arrested before transition to anaphase, during the progression to cytokinesis or even in daughter cells arising by inaccurate cell division (Figures $2 \mathrm{~h}-\mathrm{j}$ ). The various disturbances of the mitotic process have been recorded by live-imaging and are represented as filmstrips (Figures $3 a-d$ ).

During the present study, we were not able to show that cell death induced by apoptin in dividing cancer cells following 


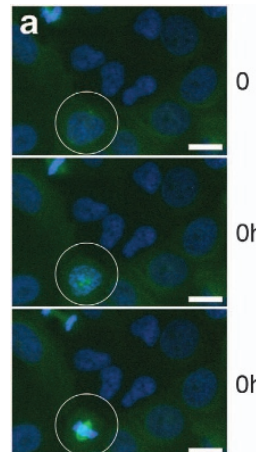

a

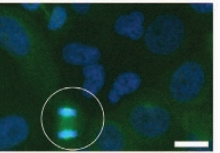

Oh32

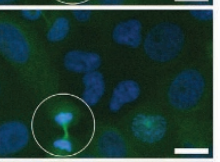

Oh38
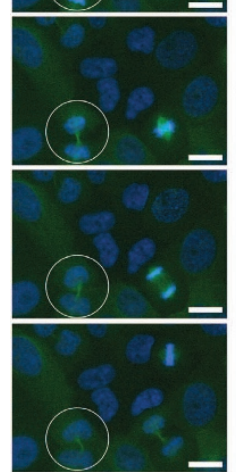

Oh56

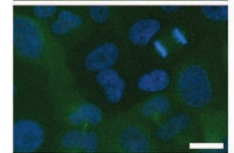

$1 \mathrm{~h} 08$

1h30

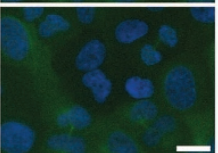

$1 \mathrm{~h} 40$

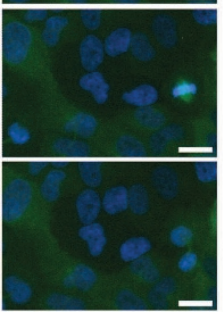

12

h44

56

2h38

3h06 b
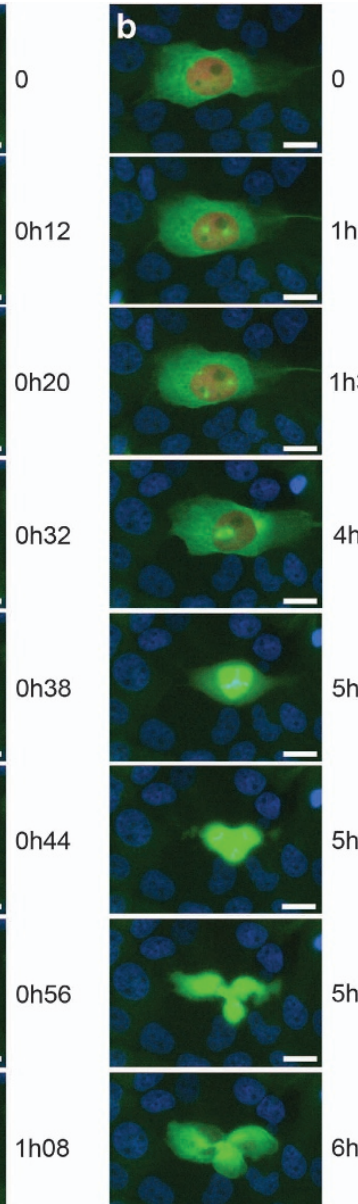
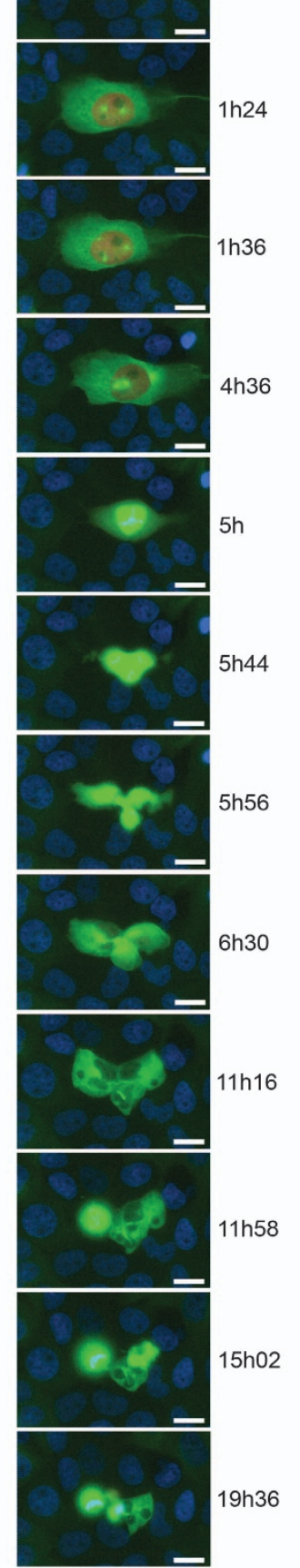
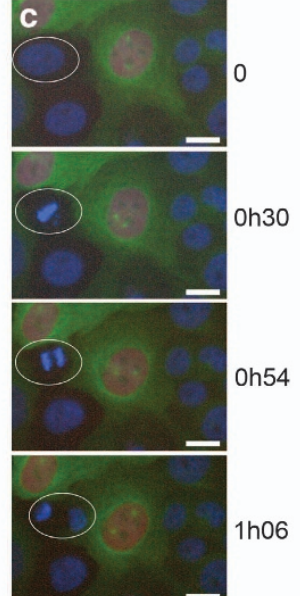

h448
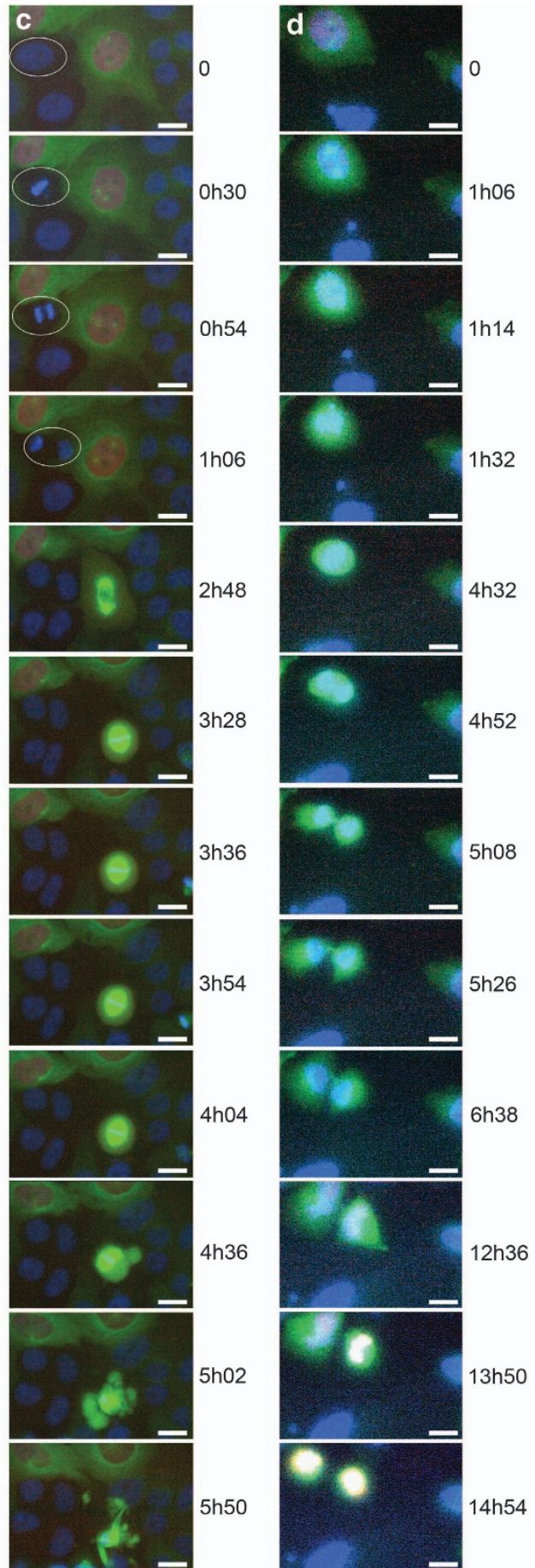

Figure 3 Dividing apoptin-positive osteosarcoma cells undergo mitotic catastrophe resulting in apoptosis. U2OS and Saos-2 cells were transiently transfected with plasmids encoding GFP-tubulin (green) and mCherry-apoptin (red), and analyzed by time-lapse microscopy. Cells were incubated with Hoechst 33342 DNA stain (blue) prior to picture capturing. Time-lapse images for blue (DNA), green (tubulin) and red (apoptin) signal were recorded for $20 \mathrm{~h}$ every $2 \mathrm{~min}$, for U2OS cells from 40-60 $\mathrm{h}$ post transfection and Saos-2 cells from $65-85 \mathrm{~h}$ post transfection. Time points of recording are indicated next to the respective images. Filmstrip (a) displays several U2OS cells transfected solely with GFP-tubulin and dividing normally ( $1 \mathrm{~h}$ to complete mitosis). One dividing cell is encircled with a white ring. Strip (b): $\mathrm{mCherry-apoptin-}$ positive U2OS cell with abnormal spindle formation completing mitosis, but undergoes apoptosis during telophase after progression through mitosis has been seriously delayed. (c) mCherry-apoptin-positive U2OS cell with normal spindle formation arrested during metaphase and eventually undergoing apoptosis during the same phase. An untransfected cell completes mitosis with normal kinetics in the same culture (white ring). In strip (d) an mCherry-apoptin-positive Saos-2 cell undergoes seemingly normal but prolonged mitosis, and apoptotic cell death occurs after cytokinesis in the two daughter cells. Scale bar: $20 \mu \mathrm{m}$. Refer to Supplementary Information to view and/or download the original movie files (avi format) 
a
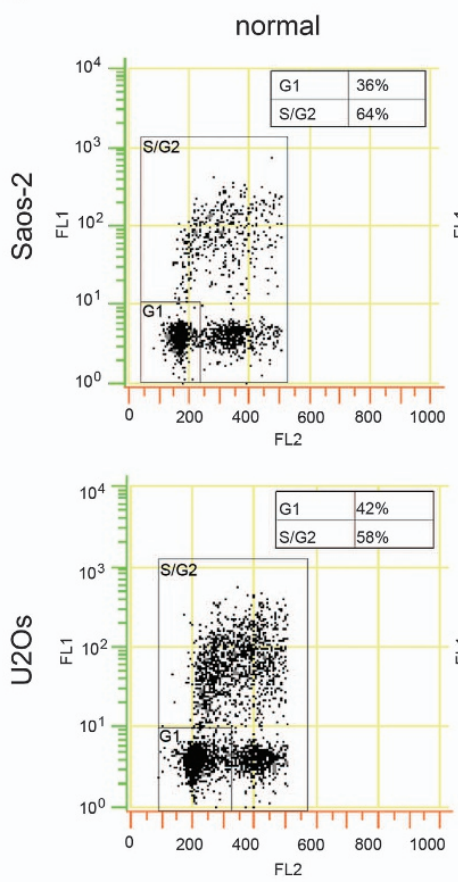

arrested
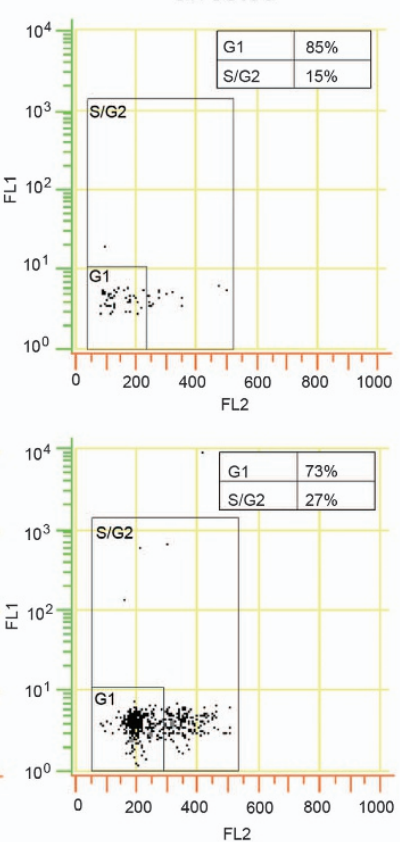

b

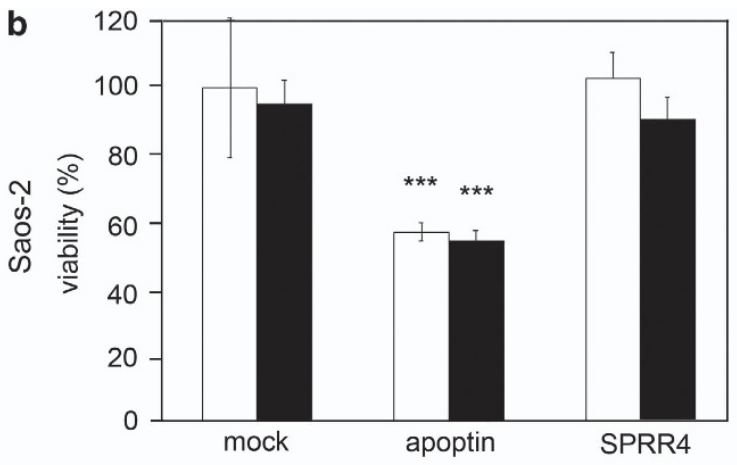

$\square$ normal $\bullet$ arrested

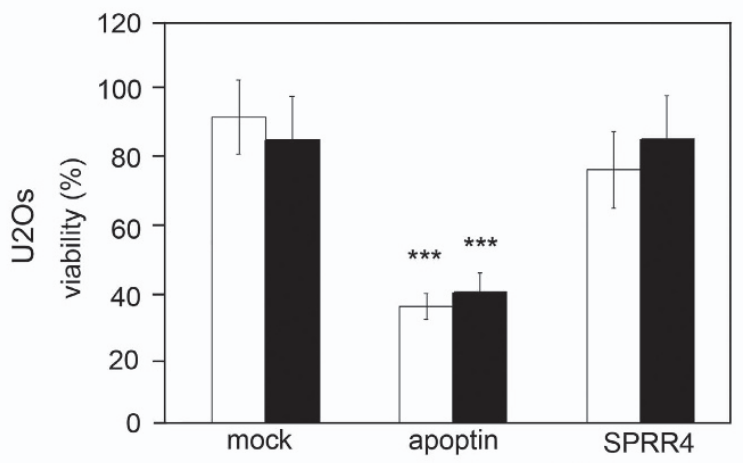

$\square$ normal arrested
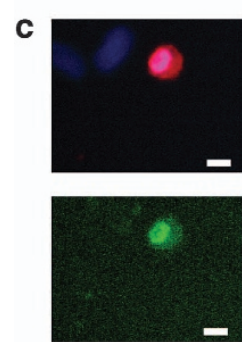

0
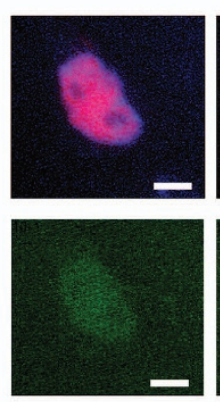

0
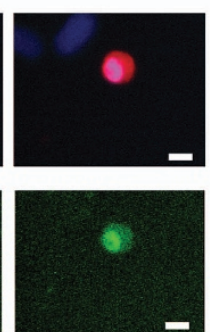

$1 \mathrm{~h} 28$
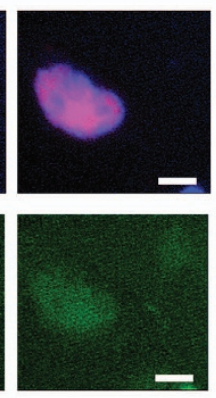

$1 \mathrm{~h} 28$
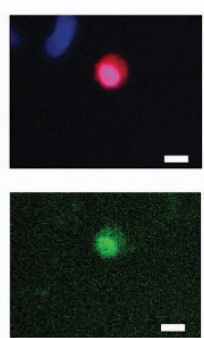

$3 \mathrm{~h} 54$
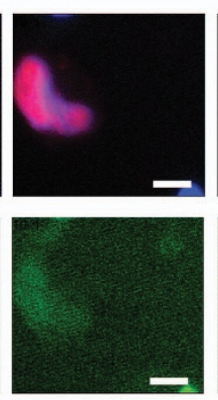

$6 h 52$
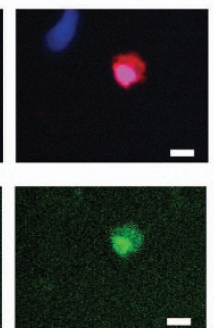

$6 \mathrm{~h} 10$
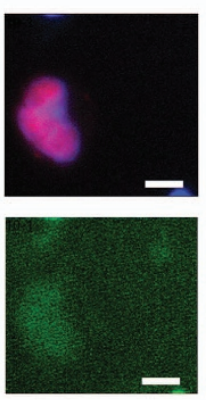

$13 \mathrm{~h} 20$
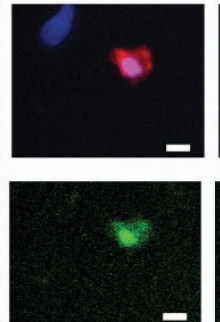

7h14
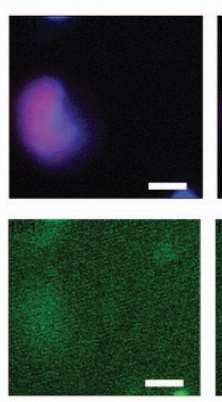

18h20

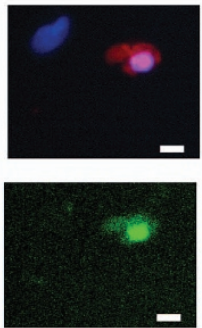

9h10
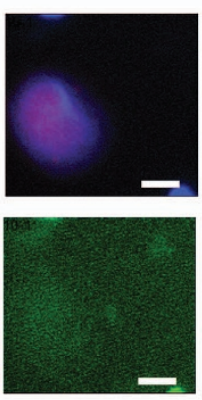

19h36

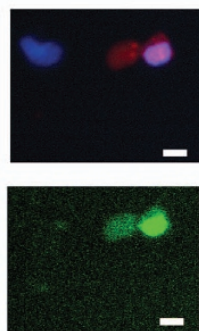

$12 \mathrm{~h} 10$
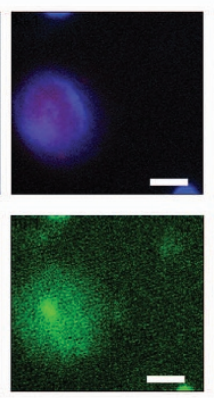

19h38

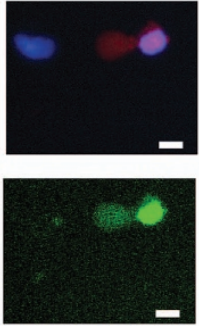

14h48
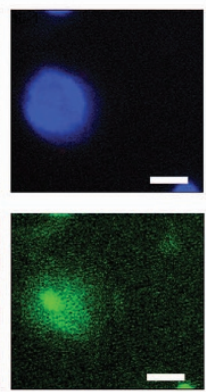

20h00

Figure 4 Apoptin induces apoptosis in cycling and arrested Saos-2 and U2OS osteosarcoma cells. Cells were either grown under cycling conditions in normal culture medium, or arrested in G1/G0 by serum starvation and mimosine treatment. Bivariate dot-plots of a FACS analysis show the log distribution of the FITC anti-BrdU staining (FL1, green fluorescence, DNA synthesis) versus the linear distribution of PI staining (FL2, red fluorescence, DNA content). Percentages of gated cells in G1/G0 and S/G2 are indicated (a). Cells were transiently mock transfected or transfected with plasmid encoding either flag-apoptin or flag-SPRR4. Viability of both normal (white bars) and arrested cells (black bars) was measured by MTT assay (Saos-2 $n=12$, U2OS $n=8$ ). Under both situations, apoptin expression resulted in a significant decrease in cell viability ( $\left.{ }^{* *}=P<0.001\right)$ (b). Cell cycle-arrested U2OS cells transfected with plasmid encoding mCherry-apoptin (red) were incubated with NucView488 caspase-3 substrate (which turns green and binds to DNA when cleaved by activated caspase-3) and analyzed by time-lapse microscopy. Cells were incubated with Hoechst 33342 DNA stain (blue) prior to picture capturing. Time-lapse images were recorded for $20 \mathrm{~h}$ every $2 \mathrm{~min} 40-60 \mathrm{~h}$ post transfection. Filmstrips from two separate movies are shown. Time points of recording are indicated below the respective images. Overall, 29 out of 37 monitored apoptin-positive cells also stained for caspase-3 activity (78\%). Scale bar: $10 \mu \mathrm{m}(\mathbf{c})$ 
induction of mitotic catastrophe was accompanied by activation of caspase-3, a marker for apoptotic cell death. However, the appearance of membrane blebbing and DNA fragmentation observed in apoptin-expressing mitotic cells (Figures $2 \mathrm{~h}-\mathrm{j}$ ) and during live-imaging (Figure 3 ) are indicative of apoptotic cell death. Similarly, the cell death induced by binding of apoptin to APC1, resulting in inactivation of APC/C, was characterized as apoptotic. ${ }^{23}$ We were able to show that apoptin-mediated cell death in non-cycling G1/G0-arrested cancer cells is associated with activation of caspase-3 (Figure 4c) in concert with our earlier work. $^{24}$

Weak inaccuracies in mitotic checkpoints are believed to be responsible for aneuploidy and genetic instability, ${ }^{20}$ which are inherent to cancer cells and drive tumor progression. ${ }^{25} \mathrm{App}$ arently, apoptin is able to sense these inaccuracies during cell division and disrupts a cancerous process by triggering mitotic catastrophe and cell death.

\section{Materials and Methods}

Cell culture. The human osteosarcoma cell lines Saos-2 (p53-null) and U2OS (p53 wild type) were purchased from the American Type Culture Collection (ATCC, Wesel, Germany) and cultured in Dulbecco's Modified Eagle's Medium (DMEM) (PAA, Diegem, The Netherlands) containing 10\% newborn calf serum (Thermo Scientific, Geel, Belgium), $100 \mu \mathrm{g} / \mathrm{ml}$ penicillin, $100 \mu \mathrm{g} / \mathrm{ml}$ streptomycin (Duchefa, Biochemie, Haarlem, The Netherlands) and $2 \mathrm{mM}$ glutaMAX (PAA). Cells were cultured at $37^{\circ} \mathrm{C}$ in a humidified $5 \% \mathrm{CO}_{2}$ incubator. Cell morphology was regularly monitored to control the absence of cross contamination.

Plasmid constructions and transfection experiments. The construction and expression of pcDNA3.1 $\left(+\right.$ ) flag-apoptin was previously described ${ }^{11}$ and contains the apoptin sequence published by Noteborn et $\mathrm{al}^{26}$ fused with a flag-tag (Life Technologies, Bleiswijk, The Netherlands) at its N-terminus. Full-length SPRR4 cloned into the pECV25 expression vector was previously described ${ }^{27}$ and provided with the same N-terminal flag-tag as apoptin. SPRR4 was chosen as a control because, in contrast to apoptin, it does not induce apoptosis (Supplementary Figure 1A) but still shares many biochemical characteristics with apoptin, that is, protein size, high proline content, isoelectric point, nuclear/cytoplasmic localization (Supplementary Figures 1B-E) and DNA binding. ${ }^{28,29}$ Plasmid encoding GFP-tubulin was purchased from Clontech (Mountain View, CA, USA). mCherry was amplified by PCR from pRSET-bmCherry ${ }^{30}$ and introduced into pcDNA3.1 (+ ) apoptin to yield a plasmid encoding apoptin $\mathrm{N}$-terminally fused to mCherry.

Cells were transfected with plasmids by using Amaxa nucleofection (Lonza AG, Cologne, Germany) according to an adaptation of the manufacturer's protocol. Briefly, $10^{6}$ cells were taken up in $120 \mu$ l nucleofector buffer $\left(90 \mathrm{mM} \mathrm{Na} \mathrm{HPO}_{4}\right.$ $\mathrm{NaH}_{2} \mathrm{PO}_{4}, \mathrm{pH} 7.2,5 \mathrm{mM} \mathrm{KCl}, 10 \mathrm{mM} \mathrm{MgCl}, 20 \mathrm{mM}$ Hepes), mixed with DNA, transferred to a transfection cuvet (VWR, Amsterdam, The Netherlands) and transfected with program D-24 or X-01 for Saos-2 or U2OS, respectively. After addition of the medium, cells were either seeded on glass coverslips or in dishes appropriate for time-lapse microscopy.

Immunofluorescence assay. Cells grown on glass coverslips were, at the indicated time points after transfection, first washed with phosphate buffered saline (PBS) and subsequently fixed for 10 min with $1 \%$ formaldehyde, 5 min with $100 \%$ methanol and 2 min with $80 \%$ acetone, all at room temperature. After airdrying, the slides were used for immunocytochemical staining or stored at $-20^{\circ} \mathrm{C}$ for further analysis. For the antibody staining, the cells were first incubated with PBS, $0.05 \%$ Tween 20 (PBS-Tween) (Sigma Aldrich, Zwijndrecht, The Netherlands), 5\% normal goat serum (NGS) (Sigma Aldrich) for $1 \mathrm{~h}$. Next, the cells were incubated with the first antibody in the same buffer for $2 \mathrm{~h}$, washed with PBS-Tween and incubated with the second antibody in PBS-Tween, 5\% NGS for $1 \mathrm{~h}$. After washing with PBS-Tween, cells were incubated 15 min with Hoechst $33358(2 \mu \mathrm{g} / \mathrm{ml})$. Stained sections were mounted using PolyMount Mounting Media (Tebu-Bio, Heerhugowaard, The Netherlands) and analyzed with a fluorescence microscope (Olympus, Zoeterwoude, The Netherlands) with the Cell P software version 2.8
(Olympus). The following antibodies were used: anti-flag (rabbit polyclonal, 1:150, Sigma Aldrich) together with a rhodamine-conjugated goat antibody $(1: 100$, Sanbio, Uden, The Netherlands), and monoclonal mouse-anti-tubulin conjugated with fluorescein isothiocyanate (FITC) (1:100, Sigma Aldrich). Mitotic cells were scored based on their tubulin and DNA staining. Apoptosis was assessed based on characteristic DNA staining. ${ }^{31}$

Cell-cycle arrest. After transfection, cells were incubated with serum-free medium for 24 (U2OS) or $48 \mathrm{~h}$ (Saos-2) and further incubated for $16 \mathrm{~h}$ with $0.5 \mathrm{mM}$ mimosine to induce G1/G0 cell-cycle arrest. Control cells grown in normal medium or arrested cells were labeled for $2 \mathrm{~h}$ with BrdU (Sigma Aldrich). Simultaneous flow-cytometric analysis of incorporated BrdU and DNA content was performed on non-transfected cells according to the procedure previously describe by Stout et $\mathrm{al}^{32}$ In short, ethanol-fixed cells were pelleted and hydrolyzed with $0.02 \%$ pepsin (Sigma Aldrich) in $2 \mathrm{~N} \mathrm{HCl}$. After neutralization, the cells were incubated for $1 \mathrm{~h}$ at room temperature with FITC-conjugated mouse-anti-BrdU (DAKO, Heverlee, Belgium) diluted $1: 20$ in PBS/0.5\% Tween 20 (PBST) with 5\% new-born calf serum. Cells were washed and resuspended in PBS containing $20 \mu \mathrm{g} / \mathrm{ml}$ propidium iodide (Sigma Aldrich) and 0.01\% RNAse A (Sigma Aldrich). Cells were analyzed on a Cell Lab Quanta flowcytometer (Beckman Coulter, Woerden, The Netherlands) for DNA content (red fluorescence) and incorporated BrdU (green fluorescence). Data was analyzed using the manufacturer's software.

Caspase-3 activation. Caspase-3 activation was monitored using NucView488 caspase-3 substrate (Biotium, Hayward, CA, USA). Cells were incubated with $0.2 \mu \mathrm{M}$ NucView488 caspase-3 substrate for 30 min prior to live-cell imaging. Upon cleavage by caspase-3, the NucView488 releases a fluorescent high-affinity DNA-binding dye (green).

Cell viability assay. Transfected cells were grown in 96-wells plates in $100 \mu$ l medium. Cell viability was assayed by incubating cells for $4 \mathrm{~h}$ with $1.2 \mathrm{mM}$ MTT (3-(4,5-Dimethylthiazol-2-yl)-2,5-diphenyltetrazolium bromide, Sigma Aldrich). After removal of the medium, crystals were resuspended in $100 \mu \mathrm{l}$ DMSO. Absorbance was measured at $560 \mathrm{~nm}$ using a Glomax multi detection system plate reader (Promega, Leiden, The Netherlands). Relative viability compared to mock transfected cells was determined.

Live-cell imaging. Transfected cells were incubated with 6.25 or $25 \mathrm{ng} / \mathrm{ml}$ Hoechst 33342 for Saos-2 or U2OS, respectively, at the indicated time points after transfection. Live-cell imaging was performed on an Olympus IX81 time-lapse microscope equipped with an MT10 lamp (Olympus) and different excitation and emission filters (Olympus). Images were captured every $2 \mathrm{~min}$ for $20 \mathrm{~h}$ with the appropriate settings for GFP, mCherry and Hoechst. Movies were analyzed with the Cell M software version 3.1 (Olympus). Results are represented as filmstrips (Figure 3) but the original movies (avi format) can be viewed from the author's website (see Supplementary Information).

Statistical analysis. Each immune fluorescence assay was performed three times. For each experiment, 100 mitotic cells were scored per group. Data sets were analyzed with the paired two-tailed Student's t-test in which apoptin-positive mitotic cells were compared to each of three negative control groups. All data are means \pm S.D.; $P$-values $<0.05$ were considered significant $\left({ }^{\star \star} P<0.01,{ }^{* \star *} P<0.001\right)$.

\section{Conflict of Interest}

The authors declare no conflict of interest.

Acknowledgements. We are grateful to Marcel Schaaf (IBL) for providing the pRSET-b-mCherry plasmid, and Hans den Dulk and Marit Arp (LIC) for technical support. This work was supported by a grant from the Dutch Royal Society of Arts and Sciences (KNAW) and by the Leiden Institute of Chemistry.

1. Galluzzi L, Vitale I, Abrams JM, Alnemri ES, Baehrecke AH, Blagosklonny MV et al. Molecular definitions of cell death subroutines: recommendations of the nomenclature committee on cell death 2012. Cell Death Differ 2011; 19: 107-120.

2. Vitale I, Galluzzi L, Castedo M, Kroemer G. Mitotic catastrophe: a mechanism for avoiding genomic instability. Nat Rev Mol Cell Biol 2011; 2: 385-392. 
3. Castedo M, Perfettini JL, Roumier T, Andreau K, Medema R, Kroemer G. Cell death by mitotic catastrophe: a molecular definition. Oncogene 2004; 23: 2825-2837.

4. Roninson IB, Broude EV, Chang B-D. If not apoptosis, then what? Treatment-induced senescence and mitotic catastrophe in tumor cells. Drug Resist Updat 2001; 4: 303-313.

5. Backendorf C, Visser AE, de Boer AG, Zimmerman RME, Visser M, Voskamp $P$ et al. Apoptin: therapeutic potential of an early sensor of carcinogenic transformation. Annu Rev Pharmacol Toxicol 2008; 48: 143-169.

6. Argiris K, Panethymitaki C, Tavassoli M. Naturally occurring, tumor-specific, therapeutic proteins. Exp Biol Med (Maywood) 2011; 236: 524-536.

7. Lanz HL, Suijker J, Noteborn MH, Backendorf C. Proteasomal insensitivity of apoptin in tumor cells. Biochem Biophys Res Commun 2012; 422: 169-173.

8. Rohn JL, Zhang Y-H, Aalbers RI, Otto N, Den Hertog J, van de Velde CJ et al. A tumorspecific kinase activity regulates the viral death protein apoptin. J Biol Chem 2002; 277: 50820-50827.

9. Sun J, Yan Y, Wang XT, Liu XW, Peng DJ, Wang M et al. PTD4-apoptin protein therapy inhibits tumor growth in vivo. Int J Cancer 2009; 124: 2973-2981.

10. Jin JL, Gong J, Ying TJ, Lu YJ, Xia JJ, Xie YY et al. PTD4-apoptin protein and dacarbazine show a synergistic antitumor effect on B16-F1 melanoma in vitro and in vivo. Eur $J$ Pharmacol 2011; 654: 17-25.

11. Zimmerman RME, Peng D-J, Lanz HL, Zhang Y-H, Danen-van Oorschot AAAM, Qu S et al. PP2A inactivation is a crucial step in triggering apoptin-induced tumor-selective killing. Cell Death Dis 2012; 3: e291.

12. Foley EA, Maldonado M, Kapoor TM. Formation of stable attachments between kinetochores and microtubules depends on the B56-PP2A phosphatases. Nat Cell Biol 2011; 13: 1265-1271.

13. Westermarck J, Hahn WC. Multiple pathways regulated by the tumor suppressor PP2A in transformation. Trends Mol Med 2008; 14: 152-160.

14. Masuda $\mathrm{H}$, Miller $\mathrm{C}$, Koeffler HP, Battifora $\mathrm{H}$, Cline MJ. Rearrangement of the p53 gene in human osteogenic sarcomas. Proc Natl Acad Sci USA 1987; 84: 7716-7719.

15. Diller L, Kassel J, Nelson CE, Gryka MA, Litwak G, Gebhardt M et al. p53 functions as a cell cycle control protein in osteosarcomas. Mol Cell Biol 1990; 10: 5772-5781.

16. Cabral A, Sayin A, de Winter S, Fischer DF, Pavel S, Backendorf C. SPRR4, a novel cornified envelope precursor: UV-dependent epidermal expression and selective incorporation into fragile envelopes. J Cell Sci 2001; 114: 3837-3843.

17. de Bruin EC, Medema JP. Apoptosis and non-apoptotic deaths in cancer development and treatment response. Cancer Treat Rev 2008; 34: 737-749.

18. Rello-Varona S, Kepp O, Vitale I, Michaud M, Senovilla L, Jemaa M et al. An automated fluorescence videomicroscopy assay for the detection of mitotic catastrophe. Cell Death Dis 2010; 1: e25.

19. Kucharski TJ, Gamache I, Gjoerup O, Teodoro JG. DNA damage response signaling triggers nuclear localization of the chicken anemia virus protein apoptin. J Virol 2011; 85 12638-12649.
20. Kops GJ, Weaver BA, Cleveland DW. On the road to cancer: aneuploidy and the mitotic checkpoint. Nat Rev Cancer 2005; 5: 773-785.

21. Musacchio A, Salmon ED. The spindle-assembly checkpoint in space and time. Nat Rev Mol Cell Biol 2007; 8: 379-393.

22. Chan YW, Chen Y, Poon RY. Generation of an indestructible cycling B1 by caspase-6dependent cleavage during mitotic catastrophe. Oncogene 2009; 28: 170-183.

23. Teodoro JG, Heilman DW, Parker AE, Green MR. The viral protein apoptin associates with the anaphase-promoting complex to induce G2/M arrest and apoptosis in the absence of p53. Genes Dev 2004; 18: 1952-1957.

24. Danen-van Oorschot AA, van der Eb AJ, Noteborn MH. The chicken anemia virus-derived protein apoptin requires activation of caspases for induction of apoptosis in human tumor cells. J Virol 2000; 74: 7072-7078.

25. Hanahan D, Weinberg RA. Hallmarks of cancer: the next generation. Cell 2011; 144 646-674.

26. Noteborn MHM, de Boer GF, van Roozelaar DJ, Karreman C, Kranenburg $O$ Vos JG et al. Characterization of cloned chicken anemia virus DNA that contains all elements for the infectious replication cycle. J Virol 1991; 65: 3131-3139.

27. Vermeij WP, Alia A, Backendorf C. ROS quenching potential of the epidermal cornified cell envelope. J Invest Dermatol 2011; 7: 1435-1441.

28. Leliveld SR, Dame RT, Mammaas MA, Koerten HK, Wyman C, Danen-van Oorschot AA et al. Apoptin protein multimers form distinct higher-order nucleoprotein complexes with DNA. Nucleic Acids Res 2003; 31: 4805-4813.

29. Vermeij WP, Florea BI, Isenia S, Alia A, Brouwer J, Backendorf C. Proteomic identification of in vivo interactors reveals novel functions of skin cornification proteins. $J$ Proteome Res 2012; 11: 3068-3076.

30. Shaner NC, Campbell RE, Steinback PA, Giepmans BNG, Palmer AE, Tsien RY. Improved monomeric red, orange and yellow fluorescent proteins derived from Discosoma sp. red fluorescent protein. Nat Biotechnol 2004; 22: 1567-1572.

31. Danen-Van Oorschot AAAM, Fischer DF, Grimbergen JM, Klein B, Zhuang S-M, Falkenburg JHF et al. Apoptin induces apoptosis in human transformed and malignant cells but not in normal cells. Proc Natl Acad Sci USA 1997; 94: 5843-5847.

32. Stout GJ, van Oosten M, Acherrat FZ, de Wit J, Vermeij WP, Mullenders LH et al. Selective DNA damage responses in murine Xpa-/-, Xpc-/- and Csb-/- keratinocyte cultures. DNA Repair (Amst) 2005; 4: 1337-1344.

Cell Death and Disease is an open-access journal published by Nature Publishing Group. This work is licensed under the Creative Commons Attribution-NonCommercial-No Derivative Works 3.0 Unported License. To view a copy of this license, visit http://creativecommons.org/licenses/by-nc-nd/3.0/

Supplementary Information accompanies the paper on Cell Death and Disease website (http://www.nature.com/cddis) 